\title{
Learning Impedance Controller Parameters for Lower-Limb Prostheses
}

\author{
Navid Aghasadeghi, Huihua Zhao, Levi J. Hargrove, Aaron D. Ames, Eric J. Perreault and Timothy Bretl
}

\begin{abstract}
Impedance control is a common framework for control of lower-limb prosthetic devices. This approach requires choosing many impedance controller parameters. In this paper, we show how to learn these parameters for lower-limb prostheses by observation of unimpaired human walkers. We validate our approach in simulation of a transfemoral amputee, and we demonstrate the performance of the learned parameters in a preliminary experiment with a lower-limb prosthetic device.
\end{abstract}

\section{INTRODUCTION}

Impedance control [1] is a common strategy for control of lower-limb prostheses [2]. It proceeds by breaking a gait cycle into four phases, and by applying a proportional derivative (PD) feedback policy within each phase [2][4]. A challenge in the application of this framework is that it requires the choice of many controller parameters, in particular 12 parameters for the knee joint, which is what we consider in this paper. Currently clinicians often choose these parameters by trial and error for each patient as noted in [2], [5], [6]. In this paper, we show how to learn impedance controller parameters in an automated way using observations of unimpaired human locomotion.

A common approach to learning controller parameters is to learn from demonstrations of an expert [7]-[15]. However, there is a challenge in applying such approaches to controller parameter learning for prosthesis, because in this particular case, there is no expert who can demonstrate what the nominal joint trajectories of an amputee should be. In other words, we do not have the amputee's gait trajectories prior to amputation. To address this problem, we develop a two step controller estimation algorithm shown in Figure 1. The first step of our algorithm is to use existing invariance in locomotion to produce joint trajectories corresponding to the locomotion of the amputee subject. To do so, we utilize locomotion trajectories that have been shown to be invariant across subjects and walking speeds [16]. By tracking the invariant trajectories on a model which uses the amputee's physical characteristics, i.e. mass and length

N. Aghasadeghi is with the Department of Electrical and Computer Engineering and T. Bretl is with the Department of Aerospace Engineering, University of Illinois at Urbana-Champaign, Urbana, IL, 61801, USA $\{$ aghasad1,tbretl $\} @$ illinois.edu. H. Zhao and A. D. Ames are with Department of Mechanical Engineering, Texas A\&M University, College Station, TX 77843, \{huihuazhao, aames\}@tamu.edu. L. J. Hargrove is with the Department of Physical Medicine and Rehabilitation, Northwestern University, Evanston, IL 60208 USA, and with the Center for Bionic Medicine at the Rehabilitation Institute of Chicago, Chicago, IL 60611 USA 1-hargrove@northwestern.edu. E. J. Perreault is with the Biomedical Engineering Department and the Department of Physical Medicine and Rehabilitation, Northwestern University, Evanston, IL 60208 USA, and with Sensory Motor Performance Program at the Rehabilitation Institute of Chicago, Chicago, IL 60611 USA e-perreault@northwestern.edu.

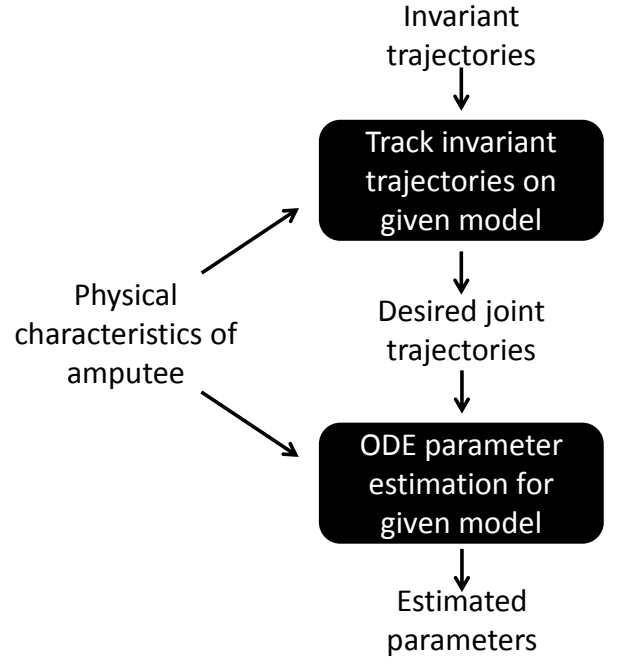

Fig. 1: Overview of the proposed algorithm.

information, we produce joint trajectories corresponding to the amputee. In the second step we use the generated joint trajectories in a model that again utilizes the amputee's physical characteristics, and is parameterized by controller parameters. We estimate controller parameters by solving a parameter estimation problem for ordinary differential equations (ODE) [17]. This leads to a computationallyefficient approach that can produce controller parameters for an amputee walking at a desired cadence, while only using pre-recorded invariant locomotion trajectories and the amputee's physical characteristics.

Biomechanical system identification is an alternative approach that in principle can be used to select impedance parameters for lower-limb impedance controllers. These approaches consist of stationary and time-varying impedance estimation techniques. In stationary impedance estimation [18], the joint under study is perturbed multiple times, and the time-invariant impedance of the joint is estimated. While these stationary estimation methods are very well studied, their applicability to the problem of locomotion controller parameter estimation is limited due to the timevarying nature of joint impedances during locomotion. Timevarying impedance estimation techniques [19] on the other hand continuously perturb the joints and can measure instantaneous impedance values in dynamic cases, i.e. when the joints are moving. However, these algorithms are often highly inefficient, requiring many perturbation experiments to be performed and averaged until an accurate measure 
of the impedance is obtained. Therefore, their applicability to measuring impedance values during locomotion is again limited. If the goal is to find parameters for prosthetic control rather than understanding the biomechanics of the intact system, it may be possible to estimate the parameters of an impedance controller that replicates the dynamics of natural locomotion, even if those parameters differ from the biomechanical properties of an intact limb.

In this paper, we apply the proposed impedance parameter learning algorithm to find controller parameters for a subject. We perform a preliminary experiment with the subject walking on a lower-limb prosthetic device, and demonstrate the results. We also validate our approach in simulation of a transfemoral amputee by showing stability through a numerical Poincare analysis [20].

The remainder of the paper is organized as follows. In Section II we introduce the hybrid system used to model the biped walker. In Section III we discuss our proposed impedance parameter estimation algorithm. Subsequently, we introduce the prosthetic simulator in section IV. We conclude by demonstrating the results in section $\mathrm{V}$, followed by a discussion in section VI.

\section{Biped Model And Control Architecture}

Bipedal walking displays both continuous and discrete behavior. It is thus natural to model bipedal walking by a hybrid system [21]. The planar point-feet biped model considered in this paper has five serial chain links (one torso, two thighs and two calves) with length and mass properties which correspond to an amputee subject. Details about the computation of mass and length values for each segment of a human subject can be found in [3], [22]. In this section, we model the dynamics of this system and introduce the impedance control framework.

\section{A. Hybrid System Model of Dynamics}

We model the dynamics of a five-link biped walker using a hybrid system. The configuration space of the biped is defined as $\mathcal{Q}=\mathbb{S}^{5}$ with coordinates $\theta=$ $\left(\theta_{s f}, \theta_{s k}, \theta_{s h}, \theta_{n s h}, \theta_{n s k}\right)^{T}$. As illustrated in Figure 2, $\theta_{1}=$ $\theta_{s f}$ is the stance foot angle, $\theta_{2}=\theta_{s k}$ is the stance knee angle, $\theta_{3}=\theta_{s h}$ is the stance hip angle which is measured from the stance thigh to the torso, $\theta_{4}=\theta_{n s h}$ is the nonstance hip angle which is measured from the torso to the non-stance thigh, and $\theta_{5}=\theta_{n s k}$ is the non-stance knee angle. Throughout the paper, a variable without a subscript denotes the vector consisting of the variable for all joints, for e.g. $\dot{\theta}=\left(\dot{\theta}_{1}, \dot{\theta}_{2}, \dot{\theta}_{3}, \dot{\theta}_{4}, \dot{\theta}_{5}\right)^{T}$.

1) Continuous Dynamics: The equations of motion of the biped are given using the Euler-Lagrange formula

$$
D(\theta) \ddot{\theta}+H(\theta, \dot{\theta})=B(\theta) u,
$$

where $D(\theta)$ is the inertial matrix and control torque distribution map $B(\theta)$ which equals the identity matrix since the biped is fully controlled, and

$$
H(\theta, \dot{\theta})=C(\theta, \dot{\theta}) \dot{\theta}+G(\theta),
$$

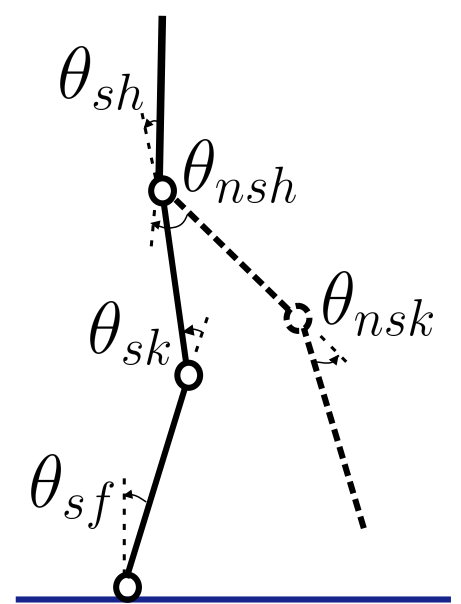

Fig. 2: Five link biped model considered in this paper and the joints angles.

2) Discrete Dynamics: The discrete dynamics are introduced to model the impact that happens instantaneously when the swing foot hits the ground. This causes an instantaneous change in the velocities of the biped and also an instantaneous switching of the stance and swing legs. We use the function $\Delta$ to denote the mapping between $\left(\theta^{T}, \dot{\theta}^{T}\right)^{T}$ just before and just after the impact. Formal definitions of the impact map can be found in [23], [24].

\section{B. Impedance Control Framework}

In this section, we describe the impedance control architecture used in control of lower-limb prosthetic devices [2]. In this framework, one gait cycle is divided into multiple phases. Within each phase, a unique impedance control for each joint is used to produce control inputs. Figure 3 demonstrates the division of a gait cycle into four phases.

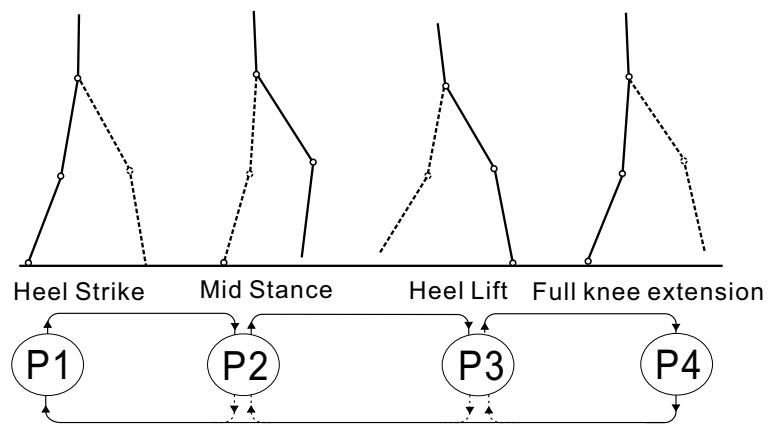

Fig. 3: Separation of gait into four phases

We consider the separation of gait into four phases, denoted $p=1, \ldots, 4$. Each phase begins at time $t_{0}^{p}$ and ends at $t_{f}^{p}$. These phases consist of

1) P1 from foot strike to mid stance denoted by passing a threshold $\theta_{s f}<t h r$ [2],

2) P2 from mid stance to foot lift [25],

3) P3 from foot lift to full knee extension (i.e. $\dot{\theta}_{n s k}<0$ ),

4) P4 from full knee extension to foot strike [25]. 
The commanded torque of an impedance controller at joint $i$ during a phase $p \in\{1,2,3,4\}$ can be represented by the following equation

$$
u_{i}^{p}(t)=k_{i}^{p}\left(\theta_{i}(t)-\theta_{i, e}^{p}\right)+b_{i}^{p} \dot{\theta}_{i}(t),
$$

where $u_{i}(t)$ denotes the input torque, $\theta_{i}(t)$ is the angle and $\dot{\theta}_{i}(t)$ denotes the angular velocity of joint $i$ at time $t$. The impedance parameters consist of

1) $k_{i}^{p}$ denoting the stiffness,

2) $b_{i}^{p}$ denoting the damping,

3) $\theta_{i, e}$ denoting the constant equilibrium angle.

These parameters are constant within each phase of the gait. We refer to the vector of all impedance parameters at joint $i$ during phase $p$ as

$$
\beta_{i}^{p}=\left[k_{i}^{p}, \theta_{i, e}^{p}, b_{i}^{p}\right]^{T} .
$$

In this paper, we will consider having impedance control only at the knee, i.e. joints $i=2$ and $i=5$ denoting the stance and swing knee, with a total of 12 parameters to be chosen for the knee. The control input at other joints is described when necessary.

Note that the impacts in the hybrid system occur only during the transitions between the phases. Therefore, within each phase $p$, the biped system evolves from time $t_{0}^{p}$ to $t_{f}^{p}$ according to the following continuous dynamics, governed by the Euler-Lagrange equations

$$
\begin{cases}D(\theta) \ddot{\theta}+H(\theta, \dot{\theta})=u & \forall t \in\left[t_{0}^{p}, t_{f}^{p}\right] \\ \left(\theta\left(t_{0}^{p}\right), \dot{\theta}\left(t_{0}^{p}\right)\right)=R\left(\theta\left(t_{f}^{p-1}\right), \dot{\theta}\left(t_{f}^{p-1}\right)\right), & \end{cases}
$$

where we let $p-1=4$ if $p=1$. The initial condition to the ODE for each phase is given by the function $\mathrm{R}$ defined as

$$
R(\theta(t), \theta(t))= \begin{cases}\Delta(\theta(t), \theta(t)) & \text { if at impact } \\ (\theta(t), \theta(t)) & \text { otherwise. }\end{cases}
$$

For joints $i=2,5$, which are controlled using an impedance controller, we replace $u_{i}=k_{i}\left(\theta_{i}-\theta_{i, e}\right)+b_{i} \dot{\theta}_{i}$ to show the dynamics as

$\left\{\begin{array}{l}k_{i}\left(\theta_{i}-\theta_{i, e}\right)+b_{i} \dot{\theta}_{i}=[D(\theta) \ddot{\theta}]_{i}+[H(\theta, \dot{\theta})]_{i} \forall t \in\left[t_{0}^{p}, t_{f}^{p}\right] \\ \left(\theta\left(t_{0}^{p}\right), \dot{\theta}\left(t_{0}^{p}\right)\right)=R\left(\theta\left(t_{f}^{p-1}\right), \dot{\theta}\left(t_{f}^{p-1}\right)\right) .\end{array}\right.$

where the notation $[v]_{i}$ denotes the $i$-th element of vector $v$.

\section{Learning Impedance Controller Parameters}

\section{A. Producing Desired Joint Trajectories for an Amputee}

A common approach to learning controller parameters is to learn from demonstrations of an expert [7]-[15]. However, these approaches can not be extended to learning prosthetic parameters, because in this particular case, there is no expert who can demonstrate what the nominal joint trajectories of an amputee should be. In other words, we do not have

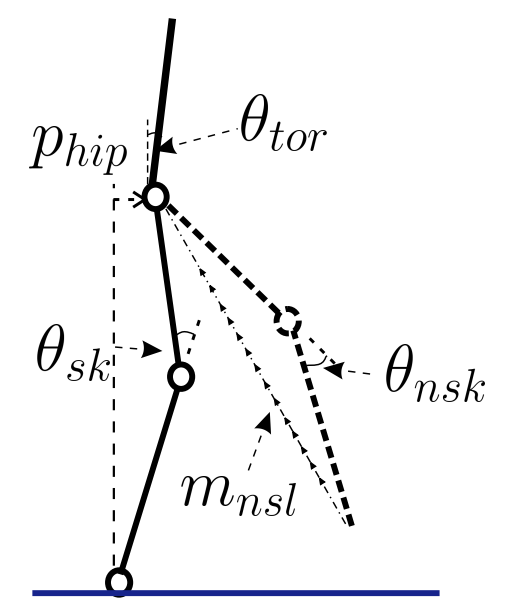

Fig. 4: The chosen invariant locomotion outputs.

the amputee's gait trajectories prior to amputation. In this section, we address this problem by using a set of locomotion trajectories that have been shown to be invariant across subjects [16] and across different walking speeds. Alternative approaches such modeling locomotion with a cost function can also be used. We proceed by incorporating the physical characteristics of the amputee, i.e. the segment lengths and masses, into our discussed biped model, and then tracking the invariant trajectories on the biped model. To track the invariant trajectories, we make use of an input/output (I/O) linearization controller [24], [26].

We use the following kinematic trajectories, which were shown to be stereotypical across subjects and walking speeds [16]:

- $z_{1}$ denoting slope of the non-stance leg $m_{n s l}$,

- $z_{2}$ denoting stance knee angle $\theta_{s k}$,

- $z_{3}$ denoting non-stance knee angle $\theta_{n s k}$,

- $z_{4}$ denoting the angle of the torso from vertical $\theta_{t o r}$,

- $z_{5}$ denoting the horizontal position of the hip $p_{h}$.

The variables corresponding to the chosen invariant trajectories are shown in Figure 4.

Note that, due to the invariance of the kinematic trajectories with respect to speed, they can be scaled in time to provide kinematic trajectories and subsequently joint trajectories for different speeds for every subject. These modulated joint trajectories can then be used to produce controller parameters leading to different walking speeds [16].

We consider observing sampled noisy observations of the invariant trajectories, and next we describe how to find their continuous estimates to use for tracking.

\section{B. Continuous Estimates of Sampled Observations}

We consider observing invariant kinematic trajectories that are sampled in time, and corrupted by noise. These observations, which are obtained from motion capture data of unimpaired human walking, are represented by $y_{i}$ for $i=1, \ldots, 5$ at time instants $t_{1}, \ldots, t_{N}$

$$
y_{i}(k)=z_{i}\left(t_{k}\right)+\epsilon_{i}(k), \quad k=1, \ldots, N .
$$


The random variables $\epsilon_{i}(k) \sim N\left(0, \sigma_{k, i}^{2}\right)$ are i.i.d and represent measurement noise. As it will be described in section III-C, to find controller parameters $\hat{\beta}$ we first need to find continuous estimates of $\hat{z}_{i}$ given observed noisy samples $y_{i}$. While in general spline functions can be fitted to observed samples to find these estimates, we fit the kinematics trajectories with the following two functions:

$$
\begin{aligned}
& g_{1}(t, \boldsymbol{\alpha})=e^{-\alpha_{1} t}\left(\alpha_{2} \cos \left(\alpha_{3} t\right)+\alpha_{4} \sin \left(\alpha_{3} t\right)\right)+\alpha_{5}, \\
& g_{2}(t, v)=v t
\end{aligned}
$$

We use the function $g_{1}$ to fit $y_{1}, \ldots, y_{4}$ and we use $g_{2}$ to fit $y_{5}$. These functions have shown to produce good fits to the chosen kinematic trajectories in different locomotion modes [27]. However, other regression approaches such as spline fitting can be used as well. The following optimizations are solved to produce estimates $\hat{z}_{i}$ for $i=1, \ldots, 4$

$$
\hat{\boldsymbol{\alpha}}_{i}=\arg \min _{\boldsymbol{\alpha}} \sum_{k=1}^{K} \frac{\left(y_{i}(k)-g_{1}\left(t_{k}, \boldsymbol{\alpha}\right)\right)^{2}}{\hat{\sigma}_{k, i}^{2}} \Rightarrow \hat{z}_{i}=g_{1}\left(t, \hat{\boldsymbol{\alpha}}_{i}\right)
$$

and for $i=5$

$$
\hat{v}=\arg \min _{v} \sum_{k=1}^{K} \frac{\left(y_{5}(k)-g_{2}\left(t_{k}, v\right)\right)^{2}}{\hat{\sigma}_{k, 5}^{2}} \Rightarrow \hat{z}_{5}=g_{2}(t, \hat{v}),
$$

where $\hat{\sigma}_{k, i}^{2}$ are variances estimated from recorded kinematic data.

These estimates of invariant trajectories are then tracked using the I/O linearization controller to produce joint trajectories $\hat{\theta}$, and numerical differentiation is used to find derivatives $\hat{\dot{\theta}}$, leading to state estimates $\hat{x}=\left[\hat{\theta}^{T}, \hat{\dot{\theta}}^{T}\right]^{T}$. The produced joint trajectories will then be used in an ODE estimation to find impedance parameters.

\section{Impedance Parameter Estimation}

As discussed in section II-B the dynamic equations for joints $i=2,5$ can be described by an ODE (5) parameterized by impedance parameters $\beta_{i}^{p}=\left[k_{i}^{p}, \theta_{i, e}^{p}, b_{i}^{p}\right]^{T}$ during each phase. If we let $x=\left[\theta^{T}, \dot{\theta}^{T}\right]^{T}$ denote the state of the system and drop the superscript $p$ for notational simplicity, the dynamic equation (5) can be represented as an implicit ODE parameterized by $\beta_{i}$

$$
\left\{\begin{array}{l}
F_{i}\left(\beta_{i}, x, \dot{x}\right)=0 \quad \forall t \in\left[t_{0}, t_{f}\right] \\
x\left(t_{0}\right)=x_{0}
\end{array}\right.
$$

where $F_{i}=[D(\theta) \ddot{\theta}]_{i}+[H(\theta, \dot{\theta})]_{i}-\left[k_{i}\left(\theta_{i}-\theta_{i, e}\right)+b_{i} \dot{\theta}_{i}\right]$.

We consider the problem of estimating unknown impedance parameters $\beta_{i}$ given the parameterized ODE (8) and observations $y(k)$.

The described problem falls in the framework of parameter estimation for ODEs. Two main estimation approaches have been developed to address this problem. The first class of methods are maximum likelihood (ML) estimators [28][31]. While these approaches have satisfactory theoretical properties, they are faced with some computational bottlenecks. These methods involve iteratively optimizing an objective function with respect to the parameter and then approximation the solution of an ODE with the current guess of the parameter. Approximating the solution of an ODE can be computationally expensive [17], [32]. Moreover due to the nonlinear nature of this optimization, the optimization often converges to a local solution [17].

Alternatively, another class of estimation approaches have been described in [17], [33], [34]. These approaches rely on a non-iterative two-step procedure. In the first step, continuous estimates of the states denoted by $\hat{x}$ and their derivatives denoted by $\dot{\hat{x}}$ are constructed using the observations $y(k)$. The estimates $\hat{x}$ are then used in the second step, which is to minimize an objective function to find the estimated ODE parameters. This approach is desirable because both steps can be implemented very efficiently. In particular spline fitting can be used to obtain consistent non-parametric estimates of the states efficiently. Subsequently a least-squares minimization can be solved to estimate the unknown parameters.

We take the two-step approach to estimating the parameters $\beta_{i}$. In section III-B we described how to obtain estimates $\hat{x}$ given observations $y(k)$. Now given the state estimates, i.e. the desired amputee joint trajectory, we solve the following least-squares minimization to find estimates of the ODE parameters, denoted by $\hat{\beta}_{i}$

$$
\hat{\beta}_{i}=\arg \min _{\beta_{i}} \int_{t_{0}}^{t_{f}}\left(F_{i}\left(\beta_{i}, \hat{x}(t), \dot{\hat{x}}(t)\right)^{2} d t .\right.
$$

Given the expression

$F_{i}=[D(\hat{\theta}) \hat{\hat{\theta}}]_{i}+[H(\hat{\theta}, \dot{\hat{\theta}})]_{i}-\left[k_{i}\left(\hat{\theta}_{i}-\theta_{i, e}\right)+b_{i} \dot{\hat{\theta}}_{i}\right]$, we can write the first two components solely as a function of the known values $\hat{x}(t)$ and $\dot{\hat{x}}(t)$, i.e. $V(\hat{x}(t), \dot{\hat{x}}(t))=$ $[D(\hat{\theta}) \ddot{\hat{\theta}}]_{i}+[H(\hat{\theta}, \hat{\hat{\theta}})]_{i}$. Moreover, if we let $U\left(\hat{x}_{N}(t), \dot{\hat{x}}(t)\right)=$ $\left[\hat{\theta}_{i}, 1, \dot{\hat{\theta}}_{i}\right]$ and we define $\tilde{\beta}_{i}=\left[k_{i},-k_{i} \times \theta_{i, e}, b_{i}\right]^{T}$ (as opposed to $\beta_{i}=\left[k_{i}, \theta_{i, e}, b_{i}\right]^{T}$ ), we can then write the minimization (9) as

$$
\hat{\tilde{\beta}}_{i}=\arg \min _{\tilde{\beta}_{i}} \int_{t_{0}}^{t_{f}}\left(V-U \tilde{\beta}_{i}\right)^{2} d t .
$$

Note that from (10), it is clear that the minimization is a linear least-squares optimization, which can be solved efficiently. We solve this optimization by discretizing the objective and solving a linear least-squares problem. Note that while we are estimating the parameters $\tilde{\beta}_{i}$ instead of $\beta_{i}$, the estimated value of $\theta_{i, e}$ can be obtained by $-\tilde{\beta}_{i}(2) / \tilde{\beta}_{i}(1)$. Thus, by first obtaining estimates of desired joint trajectories $\hat{\theta}$ for an amputee, and then solving the optimization (10), we learn impedance controller parameters for the subject.

\section{Simulating Locomotion with a Prosthetic}

In this section, we describe a simulation of a transfemoral amputee wearing a lower limb prosthetic device on one leg. The goal of designing this prosthetic simulator is to assess the stability of our impedance controller for a prosthetic device in simulation. To simulate the dynamics of our system, we utilize the aforementioned 5-link biped system 
with mass and length parameters corresponding to an average human [3], with the exception of one of the shanks, which has the physical characteristics of the Vanderbilt prosthetic leg [35]. The controllers for this simulation consist of two data-based controllers, namely the estimated impedance controller, and a stable controller replicating observed human locomotion trajectories, which we refer to as a human-inspired controller. These two controllers are described below.

\section{A. Estimated Impedance Controller}

The estimated impedance controller is used to control one knee of the 5-link biped. The control architecture is as described in section II-B, and the controller parameters are estimated using our proposed estimation method described in section III. The segment lengths of the biped corresponds to an average human [3]. Note that since the length of the prosthetic can be adjusted, we use the average human shank length for both legs, but we use different masses for the two shanks, corresponding to average human and prosthetic masses. The segment lengths are subsequently used to produce joint trajectories as described in section IIIB. Furthermore, the produced joint trajectories, along with the length and mass information are used in the estimation problem (9) to find impedance controller parameters for the biped model.

\section{B. Human-Inspired Controller}

The human-inspired controller will be used on all remaining joints of the biped, i.e. all joints except one knee joint being controlled with the estimated impedance control. While we do not know how the central nervous system controls locomotion, we can design a controller that replicates observed human trajectories. This is the motivation for using the human-inspired controller introduced in [24], which has also been implemented on the biped robot AMBER1 [36]. In short, this controller utilizes the observed locomotion trajectories $z_{i}$ described in section III-B, and fits functions to these observations with the additional constraint that the fitted functions satisfy a hybrid zero-dynamics (HZD) constraint [23], [24], [37]. Subsequently, an Input/Output linearization feedback controller [26] is used to replicate the fitted functions. The additional HZD constraint is used to ensure that the resulting fitted functions lead to a stable controller. This controller utilizes the complete information about the states of the biped system, which is not available to the prosthetic device. Thus the application of this controller in this work is limited to modeling the intact human joints.

\section{Results}

In this section we describe the results obtained through the application of the proposed controller parameter learning approach to finding impedance parameters for a subject, and we demonstrate experimental results of the subject walking with the Vanderbilt prosthetic leg [35], shown in Figure 5. We use invariant locomotion trajectories that were obtained using motion captured locomotion of unimpaired subjects.

\section{A. Prosthetic Experiment with Learned Impedance Con- troller Parameters}

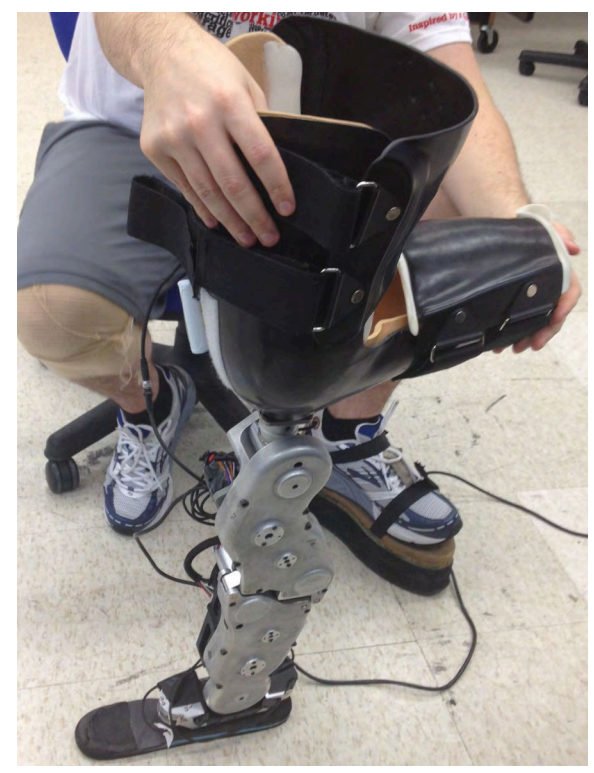

Fig. 5: Vanderbilt prosthetic leg with the attached ablebodied adapter

As a preliminary evaluation of our approach, we applied the proposed controller parameter learning algorithm to find knee controller parameters for one able-bodied subject. The ankle controller parameters were tuned experimentally by clinicians. Unimpaired subjects can walk on the Vanderbilt prosthetic leg using an able-bodied adapter [2]. We used the subject's physical characteristics, which are listed in Table I. The weight and height of the subject, along with the lengths of the thigh and shank were measured. The remaining values were obtained through the computation methods described in [3], [22]. We used averaged invariant trajectories, along with the physical characteristics of the subject to obtain controller parameters listed in Table II. The learned impedance controller parameters result in knee trajectories shown in Figure 6 for the corresponding subject. Note that these trajectories can be obtained by simulating the controlled dynamic equations with the learned impedance parameters and the subject's joint trajectories (all except the knee trajectories), which were produced in the first step of our algorithm.

To estimate controller parameters for the Vanderbilt leg, we measured the intrinsic damping of the device during stance and swing to be 0.05 and 0.08 respectively. To account for the intrinsic damping of the prosthetic, we constrained our impedance estimation to have damping values larger than the intrinsic damping of the device. We then subtracted the intrinsic damping from the estimated damping parameters, to account for the intrinsic property of the device.

The subject walked with the Vanderbilt leg, shown in Figure 5, using the learned impedance controller parameters and hand-tuned impedance parameters for the ankle. The resulting knee trajectories from multiple steps of walking 


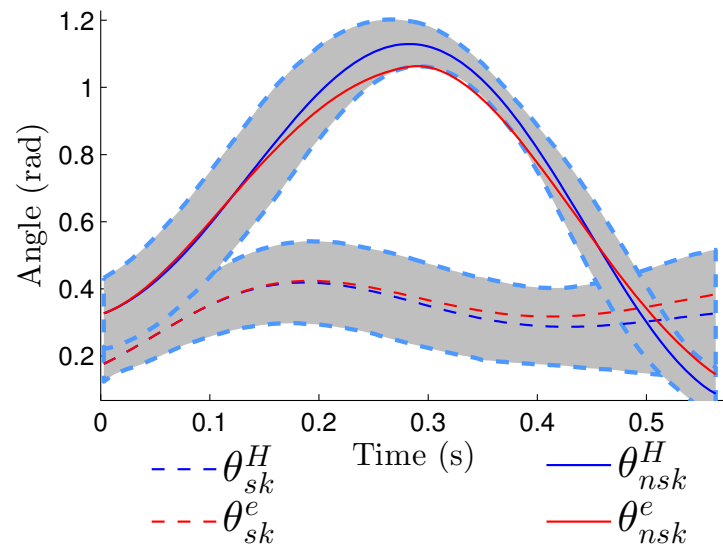

Fig. 6: The unimpaired averaged stance $\theta_{s k}^{H}$ and swing knee $\theta_{s k}^{H}$ trajectory is seen in blue, with one standard deviation shown in gray. The learned impedance parameters result in the red stance knee $\theta_{s k}^{e}$ and swing knee $\theta_{n s k}^{e}$ trajectories.

TABLE I: Table describing the mass $(\mathrm{kg})$ and length $(\mathrm{m})$ properties of the subject and of the prosthetic

\begin{tabular}{|c|c|c|c|c|c|c|c|}
\hline$*$ & $L_{\text {calf }}$ & $L_{\text {thigh }}$ & $H t$. & $m_{\text {foot }}$ & $m_{\text {calf }}$ & $m_{\text {thigh }}$ & Wt. \\
\hline subject & 0.4826 & 0.4826 & 1.7526 & 1.3812 & 4.4293 & 9.5254 & 95.25 \\
\hline prosthetic & $*$ & $*$ & $*$ & $*$ & $*$ & $*$ & 5.4431 \\
\hline
\end{tabular}

with self-selected cadence is demonstrated in Figure 7. The accompanied video shows the subject walking in the laboratory of Center for Bionic Medicine at the Rehabilitation Institute of Chicago (RIC).

Finding measures of performance for prosthetic walking is a subject of current research. In this work the resulting locomotion was evaluated by clinicians at RIC using the following measures of performance. The first measure is the smoothness of the of the knee trajectories, which can be verified from Figure 7 by the lack of any jumps at the phase transitions. Additionally, the learned prosthetic controller was evaluated by the amount of support it provided for the amputee. This was verified by the subject's ability to walk without any use of the overhead harness system and with minimal use of the hand rails.

It should be noted that the stance knee trajectories obtained from prosthetic walking in the experiment (Figure 7) have a smaller flexion angle compared to the predicted stance trajectory (Figure 6). This is commonly seen when walking with prosthetic devices [2], [38], and is not an artifact of our learned impedance parameters.

\section{B. Simulation of a Transfemoral Amputee with Learned Prosthetic Controller}

As discussed earlier, the second objective of this study was to evaluate the stability of impedance controllers for biped locomotion. We performed the simulation described in section IV with the objective of verifying the stability of the learned impedance controllers. We verified the stability using a numerical Poincare analysis [20]. The resulting
TABLE II: Estimated Controller Parameters of the Prosthesis

\begin{tabular}{|c|c|c|c|}
\hline Phase & $k[N \cdot m]$ & $b[N \cdot m \cdot s]$ & $q_{e}[$ Degree $]$ \\
\hline P1 & 4 & 0.276 & 0 \\
\hline P2 & 0.94 & 0.03 & 0.4 \\
\hline P3 & 0.29 & 0.02 & 65 \\
\hline P4 & 0.33 & 0.02 & 0 \\
\hline
\end{tabular}

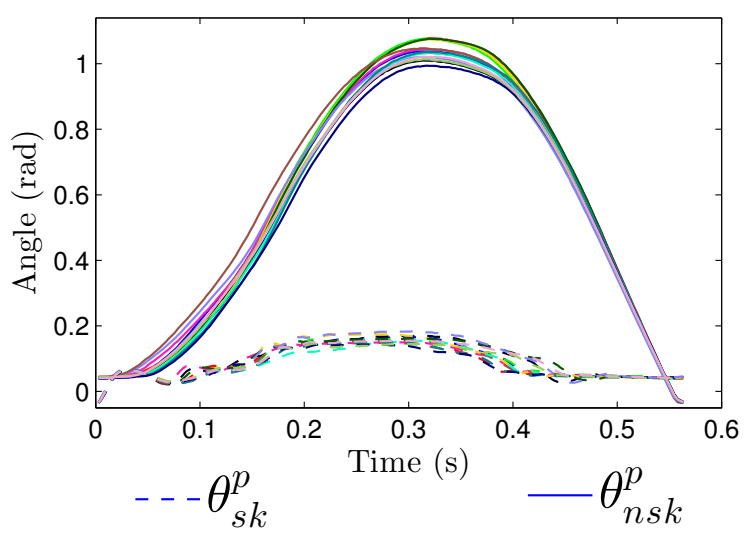

Fig. 7: The stance knee (dotted lines) and swing knee (solid lines) trajectories obtained from subject walking with learned prosthetic controller parameters.

eigenvalues all had a magnitude smaller than 0.05 , which verifies the stability of the estimated impedance control in this simulation. The simulation is also demonstrated in the accompanying video.

\section{DiscusSION AND FutURE WORK}

In this paper, we proposed a method of learning impedance controller parameters for lower-limb prostheses using only observed unimpaired kinematic trajectories, and the physical characteristics of the amputee. The main challenge in designing such a learning algorithm is that locomotion trajectories corresponding to the amputee's walking prior to amputation, i.e. the amputee's nominal joint trajectories, are often not available. Thus, we used the invariance present in locomotion trajectories along with a model that utilized the amputee's physical characteristics to track the invariant trajectories, and produce nominal trajectories for the amputee. While we applied this framework to the problem of locomotion, this idea can be used in other similar applications, where nominal observations are not available.

We would also like to emphasize that this approach is not limited to one specific dynamic model. The biped model we used was just one of several candidates of a dynamic model of a human body. Thus, while the current work has limitations due to the inconsistencies between our model and the real world, these limitations could be reduced by using a more sophisticated dynamic model. One example would be to include a dynamic model of the actuator used in the prosthetic. This should in turn improve the quality of the learned impedance parameters. Similarly, the current 
framework is not limited to estimating controller parameters only for the current control architecture.

In this work we only focused on learning controller parameters for the knee. Our future work includes learning impedance parameters for the actuated ankle as well, by incorporating a dynamic model that has feet. The experimental results presented are limited to one unimpaired subject using the able-bodied adapter. Experiments with more subjects are currently under investigation. Furthermore, it would be beneficial to include measures of performance such as symmetry between the intact and prosthetic leg, in the future. Other extensions include performing this analysis to learn impedance parameters for different locomotion modes such as stair ascent/descent and walking on ramps.

\section{ACKNOWLEDGMENTS}

This work was supported by the NSF program in Cyberphysical systems, grant \#0939963, and by the National Institute of Neurological Disorders and Stroke of the National Institutes of Health under award \#F31NS080618.

\section{REFERENCES}

[1] N. Hogan, "Impedance control: An approach to manipulation," in American Control Conference, 1984. IEEE, 1984, pp. 304-313.

[2] F. Sup, A. Bohara, and M. Goldfarb, "Design and control of a powered transfemoral prosthesis," The International journal of robotics research, vol. 27, no. 2, pp. 263-273, 2008.

[3] D. A. Winter, Biomechanics and Motor Control of Human Movement, 2nd ed. Wiley-Interscience, May 1990.

[4] D. Braun and M. Goldfarb, "A control approach for actuated dynamic walking in biped robots," Robotics, IEEE Transactions on, vol. 25, no. 6, pp. 1292-1303, 2009.

[5] F. Sup, H. A. Varol, and M. Goldfarb, "Upslope walking with a powered knee and ankle prosthesis: initial results with an amputee subject," Neural Systems and Rehabilitation Engineering, IEEE Transactions on, vol. 19, no. 1, pp. 71-78, 2011.

[6] R. D. Gregg and J. W. Sensinger, "Biomimetic virtual constraint control of a transfemoral powered prosthetic leg," in American Control Conference, Washington, DC, 2013.

[7] C. G. Atkeson and S. Schaal, "Robot learning from demonstration," in Machine Learning-International Workshop then Conference. Morgan Kaufmann Publishers, Inc., 1997, pp. 12-20.

[8] S. Schaal, J. Peters, J. Nakanishi, and A. Ijspeert, "Learning movement primitives," Robotics Research, pp. 561-572, 2005.

[9] P. Abbeel and A. Ng, "Apprenticeship learning via inverse reinforcement learning," in Proceedings of the twenty-first international conference on Machine learning. ACM, 2004, p. 1.

[10] J. Nakanishi, J. Morimoto, G. Endo, G. Cheng, S. Schaal, and M. Kawato, "Learning from demonstration and adaptation of biped locomotion," Robotics and Autonomous Systems, vol. 47, no. 2, pp. 79-91, 2004.

[11] B. Ziebart, A. Maas, J. Bagnell, and A. Dey, "Maximum entropy inverse reinforcement learning," in Proc. AAAI, 2008, pp. 1433-1438.

[12] N. Aghasadeghi and T. Bretl, "Maximum entropy inverse reinforcement learning in continuous state spaces with path integrals," in Intelligent Robots and Systems (IROS), 2011 IEEE/RSJ International Conference on. IEEE, 2011, pp. 1561-1566.

[13] B. D. Argall, S. Chernova, M. Veloso, and B. Browning, "A survey of robot learning from demonstration," Robotics and Autonomous Systems, vol. 57, no. 5, pp. 469-483, 2009.

[14] D. Nguyen-Tuong and J. Peters, "Model learning for robot control: a survey," Cognitive processing, vol. 12, no. 4, pp. 319-340, 2011.

[15] N. Aghasadeghi, A. Long, and T. Bretl, "Inverse optimal control for a hybrid dynamical system with impacts," in Robotics and Automation (ICRA), 2012 IEEE International Conference on. IEEE, 2012, pp. 4962-4967.

[16] N. A. Borghese, L. Bianchi, and F. Lacquaniti, "Kinematic determinants of human locomotion." The Journal of physiology, vol. 494, no. Pt 3, pp. 863-879, 1996.
[17] N. Brunel, "Parameter estimation of odes via nonparametric estimators," Electronic Journal of Statistics, vol. 2, pp. 1242-1267, 2008

[18] R. Kearney, I. Hunter et al., "System identification of human joint dynamics." Critical reviews in biomedical engineering, vol. 18, no. 1 , p. 55,1990

[19] J. MacNeil, R. Kearney, and I. Hunter, "Identification of time-varying biological systems from ensemble data (joint dynamics application)," Biomedical Engineering, IEEE Transactions on, vol. 39, no. 12, pp. 1213-1225, 1992.

[20] B. Morris and J. Grizzle, "A restricted poincaré map for determining exponentially stable periodic orbits in systems with impulse effects: Application to bipedal robots," in Decision and Control, 2005 and 2005 European Control Conference. CDC-ECC'05. 44th IEEE Conference on. IEEE, 2005, pp. 4199-4206.

[21] J. W. Grizzle, G. Abba, and F. Plestan, "Asymptotically stable walking for biped robots: Analysis via systems with impulse effects," vol. 46, no. 1, pp. 51-64, Jan. 2001.

[22] S. H. Scott and D. A. Winter, "Biomechanical model of the human foot: Kinematics and kinetics during the stance phase of walking," $J$. of Biomechanics, vol. 26, no. 9, pp. 1091-1104, Sep. 1993.

[23] E. Westervelt, J. Grizzle, and D. E. Koditschek, "Hybrid zero dynamics of planar biped walkers," Automatic Control, IEEE Transactions on, vol. 48, no. 1, pp. 42-56, 2003.

[24] A. Ames, "First steps toward automatically generating bipedal robotic walking from human data," Robot Motion and Control 2011, pp. 89116, 2012.

[25] S. Jiang, S. Patrick, H. Zhao, and A. D. Ames, "Outputs of human walking for bipedal robotic controller design," in 2012 American Control Conference., Montréal, Jun. 2012.

[26] S. S. Sastry, Nonlinear Systems: Analysis, Stability and Control. Springer, Jun. 1999.

[27] M. J. Powell, H. Zhao, and A. D. Ames, "Motion primitives for humaninspired bipedal robotic locomotion: walking and stair climbing," in Robotics and Automation (ICRA), 2012 IEEE International Conference on. IEEE, 2012, pp. 543-549.

[28] E. Baake, M. Baake, H. Bock, K. Briggs et al., "Fitting ordinary differential equations to chaotic data," Physical Review A, vol. 45, no. 8, pp. 5524-5529, 1992.

[29] K. Schittkowski, "Parameter estimation in systems of nonlinear equations," Numerische Mathematik, vol. 68, no. 1, pp. 129-142, 1994.

[30] M. Peifer and J. Timmer, "Parameter estimation in ordinary differential equations for biochemical processes using the method of multiple shooting," Systems Biology, IET, vol. 1, no. 2, pp. 78-88, 2007.

[31] Z. Li, M. Osborne, and T. Prvan, "Parameter estimation of ordinary differential equations," IMA Journal of Numerical Analysis, vol. 25, no. 2, pp. 264-285, 2005.

[32] J. Ramsay, G. Hooker, D. Campbell, and J. Cao, "Parameter estimation for differential equations: a generalized smoothing approach," Journal of the Royal Statistical Society: Series B (Statistical Methodology), vol. 69, no. 5, pp. 741-796, 2007.

[33] J. Varah, "A spline least squares method for numerical parameter estimation in differential equations," SIAM Journal on Scientific and Statistical Computing, vol. 3, p. 28, 1982.

[34] A. Poyton, M. Varziri, K. McAuley, P. McLellan, and J. Ramsay, "Parameter estimation in continuous-time dynamic models using principal differential analysis," Computers \& chemical engineering, vol. 30, no. 4, pp. 698-708, 2006.

[35] B. E. Lawson, A. Huff, and M. Goldfarb, "A preliminary investigation of powered prostheses for improved walking biomechanics in bilateral transfemoral amputees," in Engineering in Medicine and Biology Society (EMBC), 2012 Annual International Conference of the IEEE. IEEE, 2012, pp. 4164-4167.

[36] S. N. Yadukumar, M. Pasupuleti, and A. D. Ames, "From formal methods to algorithmic implementation of human inspired control on bipedal robots," in Algorithmic Foundations of Robotics X. Springer, 2013, pp. 511-526.

[37] K. Sreenath, H.-W. Park, I. Poulakakis, and J. W. Grizzle, "A compliant hybrid zero dynamics controller for stable, efficient and fast bipedal walking on mabel," The International Journal of Robotics Research, vol. 30, no. 9, pp. 1170-1193, 2011.

[38] S. M. Jaegers, J. H. Arendzen, and H. J. de Jongh, "Prosthetic gait of unilateral transfemoral amputees: a kinematic study," Archives of physical medicine and rehabilitation, vol. 76, no. 8, pp. 736-743, 1995. 